\title{
Stacked S-Band Slotted Waveguide Array Antenna With Very Low Sidelobes
}

\author{
Saad I. Alhuwaimel ${ }^{1}$ \\ Department of Electronic and Electrical Engineering, \\ University College London - Torrington Place \\ London, WC1E 7JE \\ United Kingdom \\ e-mail: huwaimel@,kacst.edu.sa; \\ saad.alhuwaimel@ucl.ac.uk
}

\author{
Kin-Fai Tong \\ Department of Electronic and Electrical Engineering, \\ University College London - Torrington Place \\ London, WC1E 7JE \\ United Kingdom \\ e-mail: k.tong@ucl.ac.uk
}

\begin{abstract}
A very low 3D sidelobe ( $<-21 \mathrm{~dB})$ S-band $(2.4 \mathrm{GHz})$ Slotted Waveguide Array Antenna (SWGAA) design is presented in this paper. A single waveguide SWGAA have a fan beam radiation pattern in the elevation plane. The elevation beamwidth of the SWGAA antenna can be narrowed by stacking identically designed SWGAA elements. The stacking method, simulated results and anechoic chamber measurements are presented in this paper.
\end{abstract}

Keywords-Waveguide, Slot, SWGAA, Sidelobe Level, Stacking.

\section{INTRODUCTION}

Milling slots on a wall of a rectangular waveguide allow the confined energy to escape from the waveguide. The escaped energy caused the slots to be excited and become radiating elements. This is known as Slotted Waveguide Array Antenna (SWGAA). The SWGAA relatively light weight, especially if made from aluminum, and it can handle very high power, in order of tens of kilowatts, which make it an attractive option for high power applications, such as costal surveillance and port security radars.

The use of radiating slots on waveguide walls started during the World War II. [1] and [2]. The general procedures for the slot design was first described by [1] and [2].

There are two main types of SWGAA: resonant (standing wave) and travelling wave. In the resonant type: the SWGAA is terminated by short circuit, i.e. connecting a shortening cap at the end of the waveguide. This results in a standing wave inside the waveguide. Slots are cut where the maximum electric field is located in order to have maximum current perturbation and excite the slot. Terminating the waveguide by a short circuit force the un-radiated part of the signal to be reflected and form a standing wave, minimize the power loss and hence increase the efficiency of the SWGAA. The second type is the travelling wave SWGAA where the waveguide is terminated by a matched load to absorb the wave and prevent it from reflection and forming a standing wave.

The standing wave (resonant) type of SWGAA is considered in this work. The resonant SWGAA design procedure are discussed on $[1-6]$.

${ }^{1}$ King Abdulaziz City for Science and Technology, Kingdom of Saudi Arabia P.O Box 6086, Riyadh 11442

\section{SingLe ELEMENT S-BAND SWGAA}

\section{A. Design}

WR340/WG9A waveguide is used to design a linearly polarized S-band SWGAA. The operating frequency range of this waveguide is $2.2-3.3 \mathrm{GHz}$. The waveguide has internal dimensions of $a \times b=86.36 \times 43.18 \mathrm{~mm}$ as shown in Fig. 1 . The waveguide wall thickness used in simulations was $2.03 \mathrm{~mm}$ as it is widely available commercially.

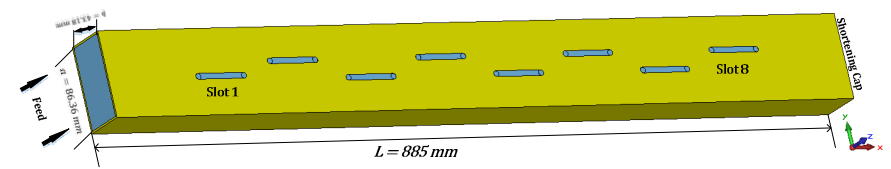

Fig. 1. Low sidelobe SWGAA

From [1] and [2], the spacing between the feeding point and the first slot and the last slot and the shortening cap is an odd multiple of quarter of the guide wavelength, as shown in Fig. 2. The wavelength of the wave propagating inside the wave guide, guide wavelength, is larger than the free space wavelength and is given by:

$$
\lambda_{g}=\frac{1}{\sqrt{\left(\frac{1}{\lambda}\right)^{2}-\left(\frac{1}{2 a}\right)^{2}}}
$$

Elliot [7] modified the slot normalized conductance proposed by [2] that is used to calculate the slots offset from the waveguide broad-wall center line. The normalized conductance is given by:

$$
\begin{aligned}
\frac{G_{\text {slot }}}{G_{\text {waveguide }}}=2.09 & \frac{\lambda_{g}}{\lambda} \frac{a}{b}\left[\cos \left(\frac{0.464 \pi}{\lambda_{g}}\right)\right. \\
& -\cos (0.464 \pi)]^{2} \sin ^{2}\left(\frac{\pi x_{1}}{a}\right)
\end{aligned}
$$

Single waveguide SWGAA with eight slots has achieved the desired azimuth beamwidth $\left(\approx 10^{\circ}\right)$. The vertical beamwidth is 
about $85.5^{\circ}$, fan beam. The uniformly distributed slots (equal offsets) around both sides of the waveguide broad-wall center line suffers from high side lobes $(\approx-13.7 \mathrm{~dB})$. The sidelobe level can be suppressed using slots tapering method around the waveguide wall center line. By trying various methods of tapering the slots distribution, it was found out that utilizing the binomial distribution when calculating each slot offset result in a very low side lobe level $(<-21 \mathrm{~dB})$. The optimized slots offsets are shown in TABLE I. Tapering the slot distribution to lower sidelobe level broaden the mainlobe $3 \mathrm{~dB}$ beamwidth by $1.3^{\circ}$. This is expected when forcing the sidelobe to go lower, more power forced to enter the main lobe.

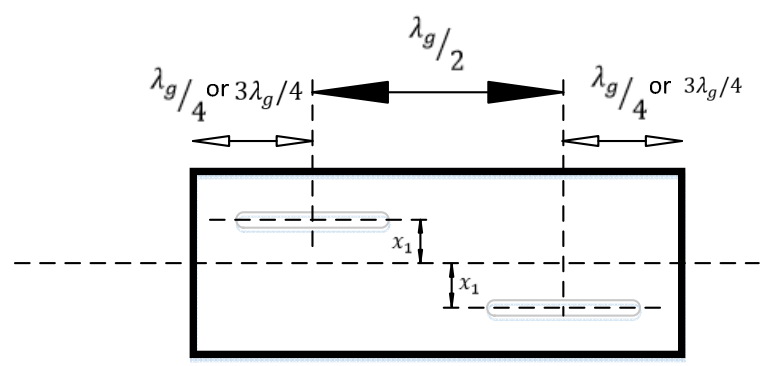

Fig. 2. Slots spacing

TABLE I. - $40 \mathrm{~dB}$ Binomial tapering coefficients and corresponding slots offsets

\begin{tabular}{|c|c|c|}
\hline Slot No. & Binomial Coffs. & Displacement (mm) \\
\hline $1 \& 8$ & 1 & 4.7 \\
\hline $2 \& 7$ & 7 & 8.2 \\
\hline $3 \& 6$ & 21 & 11.3 \\
\hline $4 \& 5$ & 35 & 13.0 \\
\hline
\end{tabular}

\section{B. Results}

The low sidelobe level SWGAA, shown in Fig. 1, was then sent out for fabrication. The reflection coefficient of the fabricated antenna has strong agreement to the simulation as shown in Fig. 3. The uniformly distributed slots SWGAA is also shown for comparison. The azimuth radiation pattern is shown in Fig. 4 of the CST [8] simulation (uniformly distributed slots and low sidelobe level designs) and anechoic chamber measurement for both co- and cross-polarization channels. As can be seen that the measured sidelobes is lower than the simulation $(<-24 \mathrm{~dB})$. Also, both $3 \mathrm{~dB}$ and $10 \mathrm{~dB}$ beamwidths are matched. The beam then broaden in the measurement after that. At this plane, the cross-polarization is very low (below - $60 \mathrm{~dB}$ ) which increase the antenna polarization purity.

The measured elevation radiation pattern shows also an excellent agreement with simulation results shown in Fig. 5. The cross polarization in this plane is also very low (below $-50 \mathrm{~dB}$ ).

\section{STACKED SWGAAS}

The azimuth beamwidth of a SWGAA is controlled by the number of slots cut on the waveguide wall. A single element SWGAA has a fan-beam in the elevation plane (E-plane). The only method to narrow the fan-beam beamwidth is by stacking identical designed SWGAA elements until the desired beamwidth is achieved. Four identical SWGAA elements, identical to the one shown in Fig. 1, were fabricated. Stacking four element was simulated with CST and gave $11.1^{\circ} \times 20.0^{\circ}$ beamwidth. The Stacked structure where then tested in an anechoic chamber.

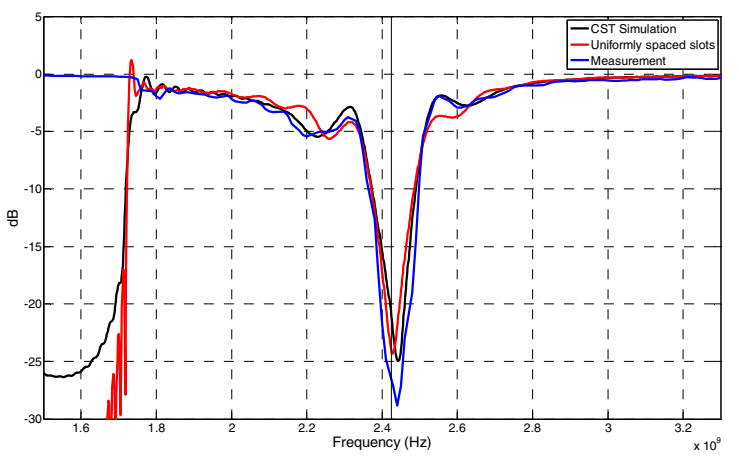

Fig. 3. Reflection coefficients for both designs

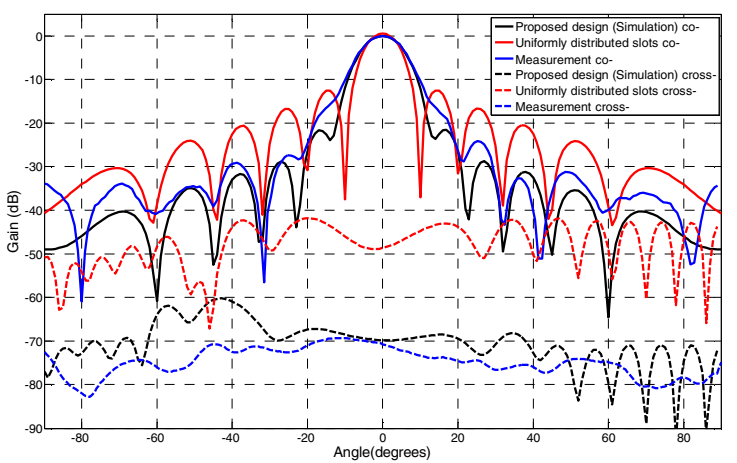

Fig. 4. Azimuth plane radiations patterns co- $\&$ cross- polarization

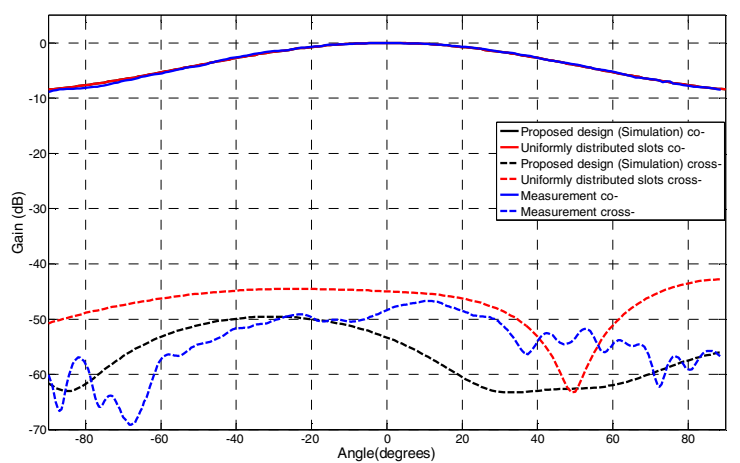

Fig. 5. Elevation plane radiation patterns co- \& cross- polarization

As each element of the SWGAAs is end-fed and because of the flange used to attach the feed, the four elements were interleaved as shown in Fig. 6 and Fig. 7. Each tow elements were fed $180^{\circ}$ out of phase from the other two.

Two other techniques were also used, in addition to the slots tapering, to lower the structure sidelobe level. First, feeding power was tapered by feeding the two side elements with $50 \%$ of the power amount fed to the two center elements. The second method was using $2 \mathrm{~mm}$ thick layers of PTFE sandwiched between each two SWGAAs to isolate them from each other. In 
addition, PTFE layers were used to isolate each SWGAA end from the flange of the adjacent SWGAA.

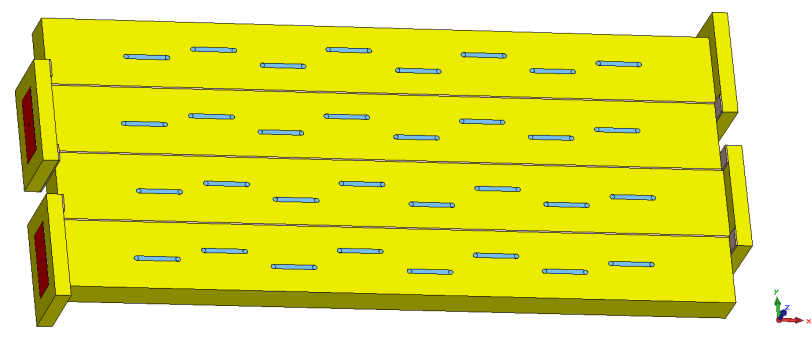

Fig. 6. Stacked SWGAAs

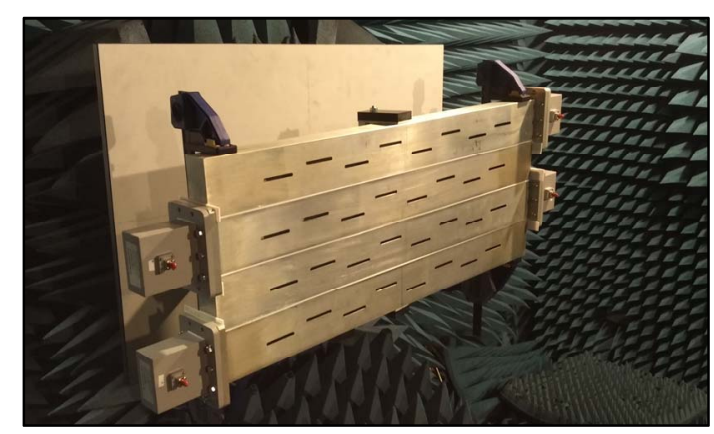

Fig. 7. Stacked SWGAAs

As shown in Fig. 8 and Fig. 9, the measurements have a good agreement to the CST simulation for both planes. The vertical $3 \mathrm{~dB}$ beamwidth slightly widened in measurements and then get wider after that. Many reasons may have contributed to that such as the human errors involved in aligning the elements when stacking, the shifts of the phase centers of each element from the adjacent elements and the losses in the cables, power splitter, attenuators and connectors.

The sidelobe level is below $-24 \mathrm{~dB}$ and the highest cross polarization point is at about $-28 \mathrm{~dB}$. That result in a very low $3 \mathrm{D}$ sidelobes with high polarization purity.

\section{CONCLUSIONS}

This work shows the possibility of achieving a low sidelobe level, high power handling and narrow beamwidth antenna using

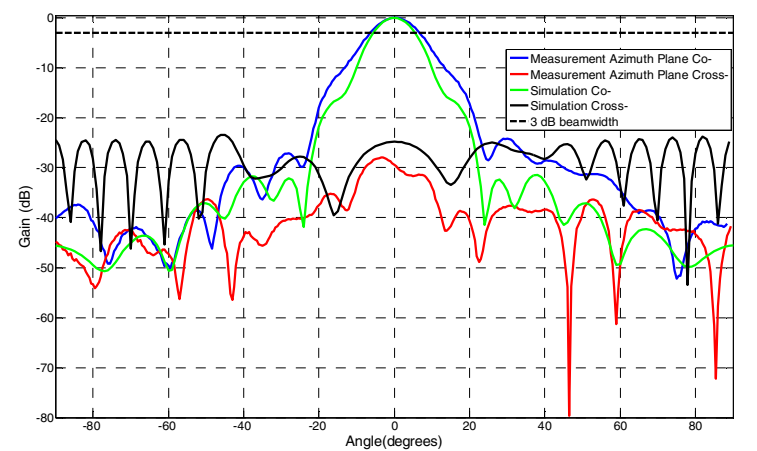

Fig. 8: Stacked SWGAAs azimuth plane radiation pattern, CST vs. measurements
SWGAAs. Methods to suppress the sidelobe level for SWGAA, both single element and stacked structure were discussed and verified both in simulation and in measurements.

The very low sidelobe level, narrow beamwidth and high polarization purity make the SWGAA an attractive option in many applications such as bistatic radars.

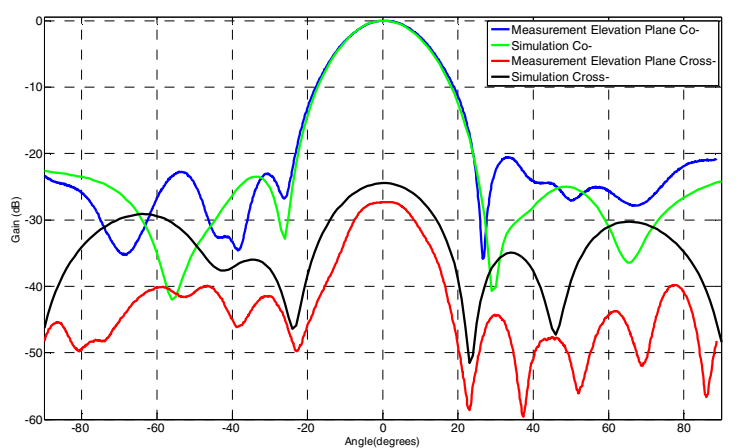

Fig. 8: Stacked SWGAAs elevation plane radiation pattern, CST vs. measurements

\section{REFERENCES}

[1] W. H. Watson, "Resonant Slots," The Journal of the Institution of Electrical Engineers - Part IIIA: Radiation, vol. 93, no. 4, pp. 747-777, 1946.

[2] A. F. Stenenson, "Theory of Slots in Rectangular WaveGuides," Journal of Applied Physics, vol. 19, no. 1, pp. 24 - 38, 1947.

[3] R. S. Elliot and L. A. Kurtz, "The design of small slot arrays," IEEE Trans. on Antenna and Propagation, vol. 26, no. 2, pp. 214-219, 1978.

[4] R. S. Elliot, "An improved design procedure for small arrays of shunt slots," IEEE Trans. on Antenna and Propagation, vol. 31, no. 1, pp. 48-53, 1983.

[5] R. S. Elliot and W. R. O'Loughlin, "The design of slot arrays including internal mutual coupling," IEEE Trans. on Antenna and Propagation, vol. 34, no. 9, pp. 1149-1154, 1986.

[6] R. S. Elliot, "Longitudinal Shunt Slots in Rectangular Waveguide: Part I, Theory," Rantecc Report, No. 72022TN-1, Calabasas, CA.

[7] R. S. Elliot, Antenna Theory and Design, New Jersy: John Wiley and Sons INC., 2003.

[8] Computer Simulation Technology - MICROWAVE STUDIO user manual, Darmstadt, Germany: CST AG, 2015. 